\title{
Simple stochastic models for glacial dynamics
}

\author{
Yosef Ashkenazy ${ }^{1}$ \\ Environmental Sciences, Weizmann Institute, Rehovot, Israel
}

Don R. Baker

Earth and Planetary Sciences, McGill University, Montreal, Quebec, Canada

Hezi Gildor

Environmental Sciences, Weizmann Institute, Rehovot, Israel

Received 22 June 2004; revised 21 October 2004; accepted 28 December 2004; published 8 February 2005.

[1] Glacial-interglacial events have several nonlinear and stochastic characteristics.

Recent studies suggested additional stochastic nonlinear features (not necessarily related to the large-scale dynamics of the glacial cycle) in the timescale of 1-100 kyr including (1) strong long-range correlations in the magnitude of climate variable increments as well as (2) a wide multifractal spectrum. Realistic climate models should reproduce these properties of the natural system. We first study several previously proposed stochastic models for glacial-interglacial dynamics and demonstrate that they do not reproduce some of the nonlinear properties of the paleoclimate proxy data. We then suggest two nonlinear stochastic models for glacial-interglacial dynamics that exhibit similar stochastic nonlinear properties to those seen in the natural data. We conjecture that interaction between fast random fluctuations (representing atmospheric variability) and slowly varying fluctuations (representing oceanic variability) may underlie the observed stochastic nonlinearity of time series for glacial-interglacial oscillations.

Citation: Ashkenazy, Y., D. R. Baker, and H. Gildor (2005), Simple stochastic models for glacial dynamics, J. Geophys. Res., 110, C02005, doi:10.1029/2004JC002548.

\section{Introduction}

[2] Climate variables, such as ice volume, temperature, and sea level, changed drastically in the last 800,000 years (800 kyr). These changes are due to successive glacialinterglacial events, each lasting approximately $100 \mathrm{kyr}$. Each glacial-interglacial oscillation consists of long glaciation periods ( $\sim 90 \mathrm{kyr})$ followed by rapid deglaciation periods ( $10 \mathrm{kyr})$. Milankovitch [1941] suggested that glacial dynamics may be related to Northern Hemisphere high latitude summer insolation; relatively cold high latitude summers will leave some snow unmelted, enabling ice growth. In addition, the following winter is relatively warm, and will have more precipitation since warmer conditions result in more precipitation. Insolation variations are attributed to changes in the eccentricity of the Earth's orbit around the Sun, to changes in the obliquity of the Earth's axis of rotation, and to changes in the precession of the Earth's orbit. These orbital parameters have timescales of $\sim 100 \mathrm{kyr}, \sim 40 \mathrm{kyr}$, and $\sim 20 \mathrm{kyr}$, respectively. Although the Milankovitch theory is widely accepted, almost all researchers agree that the eccentricity forcing with a 100 -kyr periodicity appears far too small to explain the

\footnotetext{
${ }^{1}$ Now at Department of Solar Energy and Environmental Physics, J. Blaustein Institute for Desert Research, Ben Gurion University, Sede Boker Campus, Sede Boker, Israel.

Copyright 2005 by the American Geophysical Union. 0148-0227/05/2004JC002548
}

strong 100-kyr glacial-interglacial oscillations [Imbrie et al., 1993].

[3] In the last 30 years or so, scientists debated the origin of the 100-kyr timescale of the glacial-interglacial oscillations [e.g., Kominz and Pisias, 1979; Saltzman, 1990; Imbrie et al., 1992, 1993; Hagelberg et al., 1991]. Some maintained that the "Milankovitch forcing" is the main driving mechanism of the glacial-interglacial oscillations; the strong 100-kyr cycle is the result of nonlinear rectification of the weak eccentricity (modulated by precession) variability [e.g., Berger and Loutre, 1996; Paillard, 1998]. Others suggested that the internal dynamics of the Earth climate system underlies the 100-kyr oscillations; these internal oscillations are modulated by, and phase-locked to, Milankovitch forcing [e.g., Saltzman and Sutera, 1987; Gildor and Tziperman, 2000]. Others suggested that Milankovitch forcing has only a minor effect on glacialinterglacial oscillation dynamics and that glacial-interglacial oscillations are the result of a stochastic mechanism [Kominz and Pisias, 1979; Pelletier, 1997; Wunsch, 2003; Ashkenazy et al., 2003a]. It is clear however, that whatever the role of Milankovitch forcing is, the dominant Milankovitch frequencies due to precession and obliquity explain only a relatively small portion of the variance of the proxy records while the remaining portion of the records is approximated as red noise [e.g., Kominz and Pisias, 1979; Wunsch, 2003]. By red noise, we mean that the power spectrum of the noise decays as a power law. In this paper we follow the last hypothesis and 
assume that glacial-interglacial oscillations are mainly due to internal and stochastic variability of the climate system without external insolation forcing.

[4] Different types of stochastic processes are characterized by different power spectra. The most trivial example is that of the "white noise"; the power spectrum of a Gaussian random variable is flat, indicating that the relative weight of the different frequencies is equal, similar to white color that is built from "all" colors. In a red noise spectrum the low frequencies dominate the signal; the term "red" is associated with the color red, which has a low frequency. An example of a red-noise process is a random-walk process, i.e., a process that accumulates random white noise. In that case the resulting power spectra falls like $1 / f^{\beta=2}$ where the exponent $\beta$ is the scaling exponent. In other cases the higher frequencies of the signal prevail; for example, for "blue noise" the power spectrum is more dominant for higher frequencies. Here the term "blue" also reminds us that the color blue has a high frequency. An example of "blue noise" is a derivative of white noise that has a power spectrum that is proportional to $f^{2}$; in this case the scaling exponent is $\beta=-2$ (since $\left.P(f) \sim 1 / f^{\beta}\right)$. Fractional Brownian processes yield power spectra with different scaling exponents $\beta$, indicating that a wide range of subprocesses with a wide range of frequencies control the signal [e.g., Shlesinger, 1987]. It is the scaling exponent $\beta$ which characterizes this complex situation.

[5] The red noise spectrum of the climate record may lead to the conclusion that the climate system is a simple accumulator of white noise, since the sum (i.e., integral) of white noise has a red-noise spectrum. Usually, red noise is taken as evidence of stochastic behavior; however, some deterministic models can also result in a red-noise spectrum, as shown for example by Ghil and Treut [1981]. However, recent studies indicated that glacial dynamics is not a simple linear stochastic process (such as a red noise process); in addition to their red-noise spectrum, the paleoclimate proxy records are complex and stochastically nonlinear [Lovejoy and Schertzer, 1986; Schmitt et al., 1995; Ashkenazy et al., 2003a]. By nonlinearity, we not only mean that the time series is, for example, asymmetric, but that the time series cannot be modeled by autoregression linear models. This complexity and nonlinearity is expressed by clustering of the magnitudes of increments of the series under consideration; a large magnitude increment (of the temperature change in this study) is likely to be followed by another large magnitude increment, and a small magnitude increment is likely to be followed by a small magnitude increment. In addition, the climate proxy records have a broad multifractal spectrum; that is, different moments follow different scaling laws (see Appendix A and section 2). These stochastic nonlinearities point to a specific type of mechanism that only certain types of stochastic models can reproduce. The magnitude series correlations and the wide multifractal spectrum of ice core data may shed some light on rapid climate change. Our results suggest that an episode of pronounced and rapid climate change is likely to follow a similar event. Moreover, the multifractal nature of the ice core data points to multiplicative climate processes. We note that this stochastic nonlinearity is not necessarily linked to the large-scale dynamics of the glacial cycles.
[6] Previous models addressed the red noise behavior of the proxy records of glacial-interglacial oscillations. However, these models seem not to account for the nonlinear stochastic features of the glacial-interglacial oscillations; they mainly reproduce the linear properties of the glacialinterglacial oscillations, i.e., the red-noise spectrum. Although some models have nonlinear dynamics (as reflected by the nonlinear formalism of the model), their stochastic forcing is linear; we can demonstrate that these models do not account for the nonlinear stochastic properties of the proxy records. It is fair to say that the understanding of the nonlinear stochastic nature of the glacial-interglacial oscillation dynamics remains elusive.

[7] The main objective of the present study is to propose possible conceptual mechanisms that may underlie the stochastic nonlinear features of the glacial-interglacial proxy records and can reproduce the nonlinear statistical properties of the paleoclimate data. Obviously, this objective does not result in the formation of a climate model, but instead serves the purpose of identifying some of the features that climate models must contain to successfully reproduce Earth's temperature history. A secondary objective is to reanalyze some of the previously proposed stochastic models of glacial-interglacial oscillations dynamics and verify whether they account for the recently reported stochastic nonlinear properties of long climate proxy records. There are many models that fit paleoclimate data; however, these models have completely different mechanisms (E. Tziperman, Lecture notes for Woods Hole Geophysical Fluid Dynamics summer school on conceptual models in climate dynamics, Lecture 9: Mechanisms and toy models of the glacial cycles, 2001, available at http://www.deas.harvard.edu/climate/eli/ reprints/WH_GFD_PDFvol2001.html) (hereinafter referred to as Tziperman lecture, 2001). Thus it is important to develop new tools for testing existing various glacial models and choose the more realistic ones. In this paper we suggest using the stochastic nonlinearity of paleoclimate data as an additional test for glacial models.

[8] There are several important points that we would like to discuss before describing the details of the present study: (1) The present study does not address the question of whether climate is mainly stochastic or deterministic; this question is discussed at length in the literature [e.g., Hasselmann, 1976; Kominz and Pisias, 1979; Wunsch, 2003, 2004], and we find it unnecessary to enter into this debate in our paper, and follow their conclusion that the climate system is at least partially stochastic. Our aim is to discuss only stochastic models that reproduce features of the climate system. (2) The red-noise spectrum of paleoclimate records may be produced by a deterministic system. Thus a red-noise spectrum by itself does not rule out a deterministic mechanism, but instead suggests that a simple stochastic mechanism may also be a plausible explanation for glacial dynamics. However, the evidence for stochastic nonlinearity of paleoclimate records (given by Lovejoy and Schertzer [1986], Schmitt et al. [1995], and Ashkenazy et al. [2003a]) is unlikely to be produced by deterministic glacial models, thus indicating the important role of stochastic processes in glacial dynamics. (3) It is well known that many climate models generate realistically looking glacial-interglacial time series although their mechanisms are very different [Roe and Allen, 1999] (see also Tziperman lecture, 2001). 
Thus agreement with paleoclimate data is insufficient to validate climate models. We have found additional properties of ice-age dynamics in the climate data that ideally should be reproduced by successful models, and below we discuss some generic models which exhibit similar properties.

[9] This study uses advanced statistical physics techniques to analyze paleoclimate data. We refer the reader who is interested in these techniques and terminology to Shlesinger [1987], Peng et al. [1994], Bunde and Havlin [1996], and Muzy et al. [1994], and to http://reylab.bidmc. harvard.edu/download/DFA/intro/ as well as http://www. physionet.org/tutorials/multifractal/. A nice introduction to the nonlinearity of climate records was published by King [1996] and Hagelberg et al. [1991]; note, however, that in this paper we follow the approach of Schreiber and Schmitz [2000], which also included a climate example in the their study.

[10] The paper is organized as follows. We first briefly review the evidence for the nonlinear stochastic nature of the glacial-interglacial oscillation dynamics (section 2). Then we analyze the nonlinear properties of a few stochastic models for the glacial-interglacial oscillations (section 3), demonstrating that they do not reproduce the stochastic nonlinear properties of ice core data discussed in section 2 . Next we introduce two stochastic models of glacial dynamics that reproduce the stochastic nonlinear properties of the paleoclimate proxy records (section 4). We summarize and discuss the stochastic models for glacial-interglacial oscillations in section 5 . The body of this contribution presents the important results of our study; in Appendices $\mathrm{A}-\mathrm{C}$ we summarize the statistical methods used to obtain our results.

\section{Climate Change: Periodic, Linearly Stochastic, or Nonlinearly Stochastic?}

[11] The study of the past few hundred thousand years of climate history is primarily based on proxy records of deep sea sediment cores and ice cores. Basically, heavy to light isotope ratios (like $\delta^{18} O$ and $\delta D$ ) at different depths in the cores reflect climate variables at different times. A crucial point is the depth-age relation (dating). There are several approaches used to tackle the issue of chronology: (1) Assuming that Milankovitch forcing underlies glacial dynamics, it is possible to orbitally tune the proxy records [Imbrie et al., 1984]; the tuning is usually based on the obliquity and precession parameters. (2) Build some model for sedimentation rate (or snow accumulation) to find the depth-age relation (e.g., the GT4 chronology of Petit et al. [1999]. (3) Identify extreme climate events (like ice age terminations) and then approximate their ages by assuming constant sedimentation rate and find the average time of these events [e.g., Raymo, 1997; Huybers and Wunsch, 2004]; the average times for these extreme events then provide control points for the depth-age relation. In addition, control points such as the magnetic reversal about 780 kyr ago are additional age constraints [see also Webster et al., 2004].

[12] Obviously, if the proxy records are orbitally tuned to Milankovitch forcing, it is expected that the frequency spectrum will contain the Milankovitch main periodicities. This circular approach enhances the role of Milankovitch

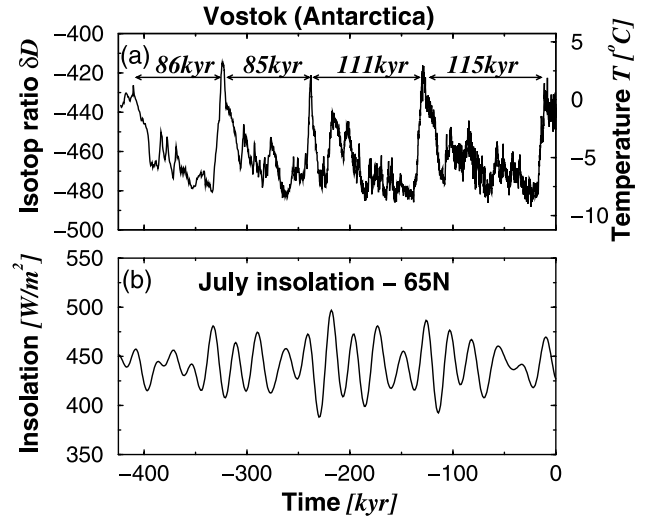

Figure 1. (a) Isotope temperature record from the Vostok (Antarctica) ice core [Petit et al., 1999] versus time (in kyr); the temperature $T$ on the right axis is linearly dependent on the hydrogen isotope ratio, $\delta D$, displayed on the left axis. The series consists of $\sim 4$ glacial cycles where the duration of each cycle is $\sim 100$ kyr: $\sim 90 \mathrm{kyr}$ of gradual cooling (glaciation) followed by $\sim 10 \mathrm{kyr}$ of rapid warming (deglaciation). (b) Summer (July) $65^{\circ} \mathrm{N}$ solar radiation as a function of age [Berger and Loutre, 1991]. The July $65^{\circ} \mathrm{N}$ insolation is dominated by the $\sim 20 \mathrm{kyr}$ precession cycle.

forcing [Neeman, 1993; Huybers and Wunsch, 2004]. On the other hand, the assumption concerning constant sedimentation rate (or an ice flow model for ice cores) is too simple to accurately determine the depth-age relation. In addition, the third approach presented above usually produces large error bars for the timing of terminations such that exact timing for the climatic events is not accurately known. Therefore, unfortunately, an accurate frequency spectrum of the climatic record is not yet available, and thus it is more difficult to evaluate the role of Milankovitch forcing on climate dynamics. Nonetheless, examining highresolution data that are not orbitally tuned is the most suitable technique to study the role of Milankovitch forcing on climate.

[13] In 1999, high-resolution ice core data from Vostok (Antarctica) became available [Petit et al., 1999]. These ice core data are longer than $3 \mathrm{~km}$ and extend back more than $420 \mathrm{kyr}$. Some of the proxies (e.g., $\delta D$ ) are sampled every meter and thus result in more than 3000 data points available for time series analysis. The depth-age relation of the Vostok record (GT4) is based on an ice-flow model and is minimally orbitally tuned. In Figure la we present the Vostok $\delta D$ record which is a proxy for local temperature during ice deposition. The record shows the last four glacial cycles with an average period of $\sim 100 \mathrm{kyr}$. We also show the corresponding $65^{\circ} \mathrm{N}$ July insolation (Figure 1b) which, according to Milankovitch theory, underlies glacial dynamics. It is clear that there is no direct (linear) relation between the two [Imbrie et al., 1993]. In Figure 2 we show the power spectrum of the ice core data (Figure 1a) and that of July $65^{\circ} \mathrm{N}$ insolation (Figure 1b). The power spectrum of the ice core data shows a pronounced peak at the 100-kyr timescale, with secondary magnitude decreasing peaks at 40-kyr, 28-kyr, 22-kyr, and 18-kyr timescales. On the other hand, the power spectrum of the insolation data does not show the most pronounced 100-kyr peak, and unlike the ice core data, 

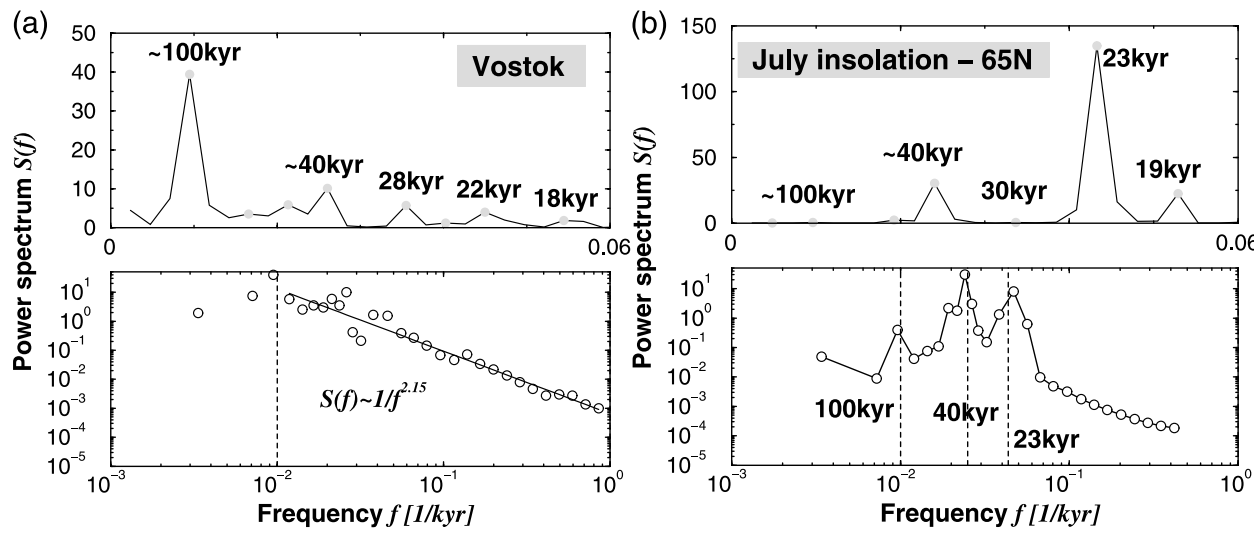

Figure 2. (a) The power spectrum of the Vostok ice core data shown in Figure 1a. (top) Linear plot. (bottom) Log-log plot (double logarithmic plot). A double logarithmic plot is usually used to estimate the exponent of a power law function. The estimated exponent is $\beta \approx 2.15$. The linear plot shows a dominant $\sim 100 \mathrm{kyr}$ cycle with secondary peaks of $\sim 40 \mathrm{kyr}$ to $\sim 20 \mathrm{kyr}$ cycles. On the other hand, the log-log plot of the power spectrum shows a red-noise-like spectrum for frequencies $1 \mathrm{kyr}^{-1}>f>0.01 \mathrm{kyr}^{-1}$ with a crossover to white noise spectrum for lower frequencies $\left(f<0.01 \mathrm{kyr}^{-1}\right)$ suggesting that the glacialinterglacial process is mainly stochastic. (b) Same as Figure $2 \mathrm{a}$ for the $65^{\circ} \mathrm{N}$ July insolation [Berger and Loutre, 1991] shown in Figure 1b. Here, unlike the power spectrum of Vostok ice core data, the $\sim 20 \mathrm{kyr}$ precession cycle is the dominant one while the $\sim 100 \mathrm{kyr}$ eccentricity periodicity is very small. Moreover, unlike Figure 2a, the log-log plot of the power spectrum still shows the dominant peaks observed in the top panel and a red-noise, power law relation is not present. Note that in the log-log plots the $x$ axis has logarithmic binning (i.e., the $x$ axis is equally spaced on logarithmic scale) to assure good estimation of the power law exponent.

the 40-kyr peak is smaller than that of the 20-kyr peaks. This dissimilarity led scientists to the conclusion that the climate system does not respond linearly to changes in insolation [e.g., Saltzman, 1990; Imbrie et al., 1992, 1993].

[14] In Figure 2 (bottom panels) we show the power spectra on a log-log plot; in a log-log plot the $x$ and $y$ axes are replaced by $\log x$ and $\log y$, and thus a power law function $1 / f^{\beta}$ appears linear on a log-log plot where the slope of this line is the exponent $\beta$. In the case of the ice core data, the power spectrum has a power law spectrum, $S(f) \sim 1 / f^{\beta}$, for frequencies larger than $1 /(100 \mathrm{kyr})$ with an exponent $\beta=2.15$; this exponent is equivalent to the detrended fluctuation analysis (DFA) exponent of $\alpha=$ $(\beta+1) / 2 \approx 1.6$ (Appendix $C$ ). The periodicities which seem to be significant on a linear plot look weak and insignificant on a log-log plot [Kominz and Pisias, 1979; Wunsch, 2003]. A power spectrum that follows a power law is a possible indication of a stochastic underlying process [Mandelbrot and van Ness, 1968]. For example, a random walk (which is a simple sum of white noise) has a red noise power spectrum $S(f) \sim 1 / f^{2}$, i.e., $\beta=2$. Consequently, one might conclude that climate change, as reflected in the Vostok ice core data, is a simple random-walk process where the increments are simple linear white noise; however, previous studies demonstrated that the ice core data are stochastically nonlinear and not a simple random-walk process [Ashkenazy et al., 2003a].

[15] The power spectrum of the insolation data plotted on a log-log plot (Figure 2b, bottom panel) does not follow scaling laws while the peaks of obliquity and precession are still present. The red-noise behavior of the ice core data and the inconsistency with the insolation data are what led other researchers [e.g., Kominz and Pisias, 1979; Wunsch, 2003] and ourselves to the con- clusion that the underlying glacial process may be mainly stochastic while the relative changes in isolation (as reflected by Milankovitch forcing) may be secondary [Kominz and Pisias, 1979; Pelletier, 1997; Wunsch, $2003,2004]$. In this work we concentrate on the stochastic properties of paleoclimate records and neglect the contribution of the Milankovitch forcing.

[16] The power spectrum reflects the two-point correlations of the time series under consideration; the information that is stored in the Fourier phases is ignored. The Fourier phases reflect information about the high-order statistics of the series and are related to the nonlinearity of the time series. Several studies analyzed the nonlinear properties of paleoclimate time series including the asymmetry of the time series [Hagelberg et al., 1994; Schreiber and Schmitz, 2000], the harmonics in the power spectrum [King, 1996], modulations of the series [King, 1996], the phase relations between different frequencies [Hagelberg et al., 1994; King, 1996], and the presence of combination tones in the power spectrum [Ghil and Treut, 1981; Yiou et al., 1994].

[17] However, the term nonlinearity is defined in different ways by different people. For example, some define nonlinearity according to the response of the system to external perturbation: If the response is linear, then the system is linear; otherwise the system is considered to be nonlinear. Others would define a system to be nonlinear according to its dynamical equations: If the system's dynamical equations contain nonlinear terms, it is considered to be nonlinear. Others refer to nonlinear dynamics when they want to describe the complex behavior of systems such as chaotic systems.

[18] In some sense, it is obvious that climate dynamics is nonlinear due to the sawtooth pattern of glacial-interglacial 


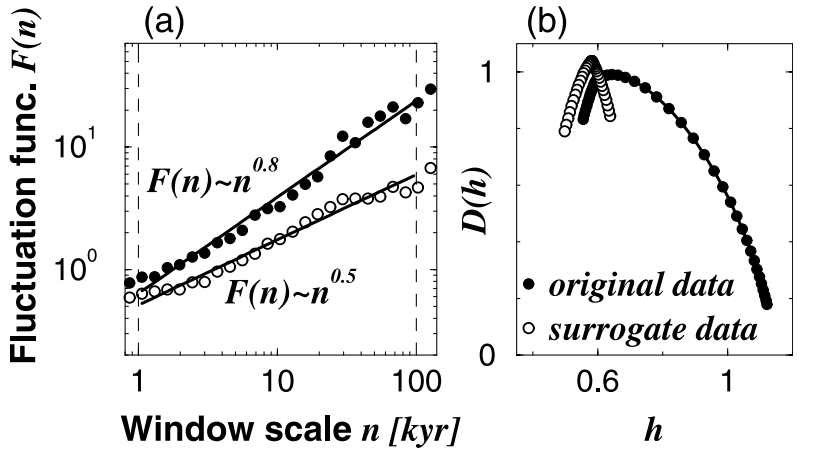

Figure 3. (a) Root mean square fluctuation (Appendix C), $F(n)$, for the magnitudes, $\left|\Delta T_{i}\right| \equiv\left|T_{i+1}-T_{i}\right|$, of the temperature increments of Vostok ice core data shown in Figure 1a [after Ashkenazy et al., 2003a]. Correlations in the magnitudes $\left|\Delta T_{i}\right|$ (solid circles) are an indication of stochastic nonlinearity of the original temperature time series $T_{i}$. The correlations in the magnitude series $\left|\Delta T_{i}\right|$ follow a scaling law $F(n) \sim n^{0.8}$ and indicate clustering of the magnitudes of temperature increments; i.e., a large $|\Delta T|$ tends to follow a large $|\Delta T|$ and a small $|\Delta T|$ tends to follow a small $|\Delta T|$. To validate the nonlinearity of the temperature increments series, a test for nonlinearity was applied after which the Fourier phases become random while the probability distribution and the power spectrum of the temperature increment series remains almost unaffected (Appendix B). Unlike the original Vostok ice core, the magnitude series $\left|\Delta T_{i}\right|$ of the phase randomized increment series is uncorrelated (open circles) with an exponent $\alpha=$ $0.5 \pm 0.05$. (b) The multifractal spectrum $D(h)$ of the Vostok data shown in Figure 1a [after Ashkenazy et al., 2003a]. The multifractal spectrum is an additional measure for nonlinearity: A wide multifractal spectrum indicates a strong nonlinearity of the series while a narrow multifractal spectrum indicates a weaker nonlinearity. The multifractal spectrum of the original Vostok data is wide (solid circles) and thus indicates a nonlinearity of the original series. After applying a surrogate data test for nonlinearity, the multifractal spectrum becomes significantly narrower (open circles) and thus confirms that multifractality is a measure for nonlinearity.

oscillations (Figure 1a). However, the nonlinearity we discuss is not related to this asymmetry, but only to the Fourier phases. Briefly, we define a time series to be linear if its statistical properties are invariant under randomization of the Fourier phases; that is, the statistical characteristics of a linear series depend solely on the power spectrum and the histogram but are independent of the Fourier phases [Schreiber and Schmitz, 1996, 2000]. The statistical properties of nonlinear time series depend on the Fourier phases. The histogram of the increment series $\left(\Delta x_{i}=x_{i+1}-x_{i}\right)$ reflects the asymmetry of the time series; a symmetric time series will have a symmetric histogram for its increment series. Because our definition for nonlinearity is related just to the Fourier phases and not to the histogram, the asymmetry of a time series is not related to the nonlinearity we discuss here. Note that the above does not imply that the asymmetry of a time series is always independent of the Fourier phases.
[19] Recently, an additional type of nonlinearity was identified in ice core data from Antarctica and Greenland based upon the discovery that the magnitudes of climate change are correlated [Ashkenazy et al., 2003a]. Given a time series $x_{i}$, the long-range correlations of the magnitude series $\left|x_{i+1}-x_{i}\right|$ reveal that the magnitude series is "clustered" [Ashkenazy et al., 2001, 2003b]. When the magnitude series is uncorrelated, the magnitudes appear as white noise without any ordering, while in correlated magnitude series the magnitude series appear in clusters of large and small magnitudes; see Figure A1 in Appendix A. It follows that a large magnitude of climate change is likely to be followed by another large magnitude of climate change, while a small magnitude is likely to be followed by a small magnitude. The nonlinear measure of magnitude series correlations may be assessed using the surrogate data test for nonlinearity (Appendix B). In this test, different series that have the same power spectrum and the same probability distribution as the data are generated while the Fourier phases of the surrogate data are random. Thus the nonlinearity of a time series that is based on the Fourier phases is destroyed after applying the surrogate data test for nonlinearity. If, indeed, long-range correlations in the magnitude series indicate the nonlinearity of the time series under consideration, they should not be present in the surrogate linearized time series. In Figure $3 a$ we show the root mean square fluctuation function (calculated using the second-order detrended fluctuation analysis, Appendix C) of the surrogate linearized data of the Vostok ice core; the magnitude series of the surrogate data is uncorrelated white noise with an exponent $\alpha=0.5 \pm 0.05$, while the magnitude series of the original Vostok data is highly correlated with an exponent of $\alpha \approx 0.8$ (we obtain similar exponent values from other ice cores [Ashkenazy et al., 2003a]). The standard error of the slope indicating the exponent value of the ice core data is 0.02 . This test demonstrates that long-range correlations in the magnitude series reflect the nonlinearity of the original time series.

[20] Previous studies indicated that ice core data from Greenland display a broad multifractal spectrum [Lovejoy and Schertzer, 1986; Schmitt et al., 1995]. The multifractal spectrum reflects the relation between the different moments of the time series; the different moments of monofractal (linear) series depend linearly on the second moment while those of multifractal time series are nonlinearly related to each other (Appendix A). In a recent study, we demonstrated that ice core data from Antarctica display a broad multifractal spectrum as well, where in this case most of the multifractal broadness comes from the negative moments [Ashkenazy et al., 2003a]; see Figure 3b. These broad spectra are an additional measure of nonlinearity since a linear series (which follows a scaling law and whose distribution does not have broad tails) has a narrow multifractal spectrum. Applying the surrogate data test to these series followed by analysis of the spectrum, we find that the linearized surrogate data multifractal spectrum is much narrower, indicating that the multifractal spectrum also reflects the stochastic nonlinearity of the ice core data.

[21] The above observations suggest that ice age dynamics is not a result of a linear process but rather has nonlinear stochastic characteristics. The origin of these stochastic nonlinear properties still remains unclear. The new measures of nonlinearity described above indicate the need to reana- 
lyze existing stochastic models for glacial-interglacial dynamics to investigate whether or not they produce synthetic climate histories with the same statistical properties as measured in the ice cores. Below we investigate a series of recent climate history models and demonstrate that they do not account for the stochastic nonlinear properties of the paleoclimate proxy data. We then suggest two possible abstract models that could underlie the stochastic nonlinearity of the ice core data. Ideally, these models should reproduce the two-point correlations (as reflected by the power spectrum), the magnitude series correlations, and the shape and broadness of the multifractal spectrum.

\section{Models for Glacial Dynamics With Linear Stochastic Forcing}

[22] In this section we review a few published models for glacial dynamics that reproduce some aspects of the statistical properties characterizing the ice core proxy records. We reanalyze these models and show that they do not account for the stochastic nonlinear properties of the ice core data described above.

\subsection{ARMA Model}

[23] The red-noise spectrum of the paleoclimate proxy records may indicate that climate change is a simple integrator of random changes in climate. Pelletier [1997] suggested that changes in global temperature $T$ over timescales of several thousands of years up to millions of years follow

$$
\frac{d T}{d t}=-\gamma T+\eta_{t},
$$

where $\eta_{t}$ is a Gaussian white noise and $\gamma T$ is a damping term which keeps the model's output from growing to infinity. The power spectrum $S(f)$ of this equation is

$$
S(f) \sim \frac{1}{\gamma^{2}+(2 \pi f)^{2}} .
$$

Note that this process is independent of the Fourier phases and just depends on the power spectrum; substituting random phases instead of the original Fourier phases will produce similar dynamics. Thus this model is linear. On the basis of physical arguments Pelletier [1997] suggested that $\gamma=1 /(40 \mathrm{kyr})$; the power spectrum has a red-noise spectrum $S(f) \sim 1 / f^{2}$ for large frequencies $f>\gamma$ and has a white noise spectrum for small frequencies $f \ll \gamma$. This model reproduces the power spectrum of paleoclimate records, i.e., red-noise spectrum for high frequencies, $f>1 /(100 \mathrm{kyr})$, and white noise (flat) spectrum for low frequencies, $f<$ $1 /(100 \mathrm{kyr})$. However, this model does not account for the following important properties of the ice core data: (1) The nonlinearity of the ice core data described above (section 2) and (2) the asymmetry (long glaciations followed by rapid deglaciation) of the ice core data.

[24] More advanced Auto Regression Moving Average (ARMA) models [Wunsch, 2003] have similar drawbacks. An ARMA process is defined as

$$
x_{n}=\sum_{i=1}^{p} a_{i} x_{n-i}+\eta_{n},
$$

where $\eta_{n}$ is Gaussian white noise. The ARMA process is a linear process. In Figure 4 we show one realization of an

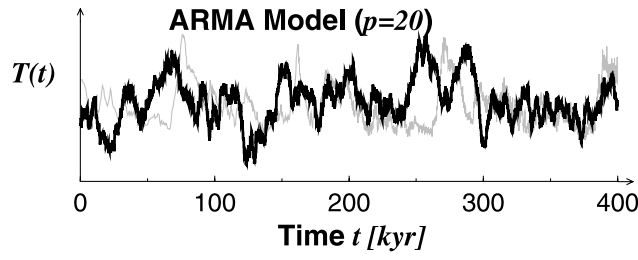

Figure 4. An example of an auto regression moving average (ARMA) process of order 20; we use the Vostok ice core data to estimate the model's parameters. The simulated time series (black curve) has the same power spectrum as seen in the Vostok data. However, it does not show the asymmetry of long glacial periods followed by short interglacial periods observed in the Vostok data (shaded curve), and it does not exhibit correlations in the magnitudes of the series' increments. Note that an ARMA process is by definition a linear process (i.e., its parameters depend solely on the power spectrum but are independent of the Fourier phases).

ARMA process with a relatively large number of coefficients, $p=20$. The model does not display the asymmetry and repetitive glacial periods of the ice core data. Moreover, as expected from a linear model, the magnitude series of the model's output $\left|x_{n+1}-x_{n}\right|$ is uncorrelated (exponent of $0.5 \pm$ 0.05 ) and the series $x_{n}$ is monofractal; therefore the stochastic nonlinear properties of long-range correlations of the magnitude series of the Vostok ice core data are not reproduced.

\subsection{Random Walk Plus Thresholds}

[25] A slightly more advanced model for ice-volume dynamics was suggested recently by Wunsch [2003]; the goal of this model was to show that it is possible to visually replicate a realistic time series for ice volume (that represents climate) by a simple stochastic model. The Wunsch model can be summarized as follows. Ice volume $V(t)$ builds up randomly to a specified maximal threshold at which point it breaks up rapidly and the volume decreases to 0 . Then, growth begins again. The ice volume thus fluctuates between the maximal threshold and zero and the ice-volume growth curve is similar to the growth of a simple linear random walk. In the Wunsch model the (maximal) threshold is subject to relatively small random fluctuations; here we used a simplified version of this model in which the threshold is fixed. We do not expect the results presented here to be qualitatively different since the model's dynamics is dominantly linear, until the ice volume hits the thresholds. A realization of this model (where the maximal threshold is constant) is shown in Figure 5. This model shows the asymmetry of the ice-volume data, which is one of the main characteristics of the glacial dynamics. However, contrary to the proxy data for ice volume, the model exhibits long periods with very low ice volume (high temperature).

[26] The model described above is a piecewise linear model; the growth of the ice volume is a simple random walk, whereas the point at which the ice volume attains the maximal ice-volume threshold and drops to zero is highly nonlinear. Since the nonlinearity of the natural data (discussed above) concerns the clustering of the magnitude 

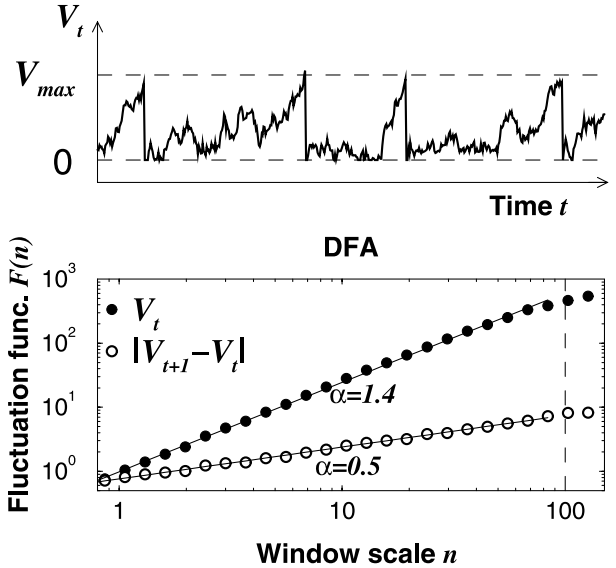

Figure 5. (top) A realization of a model for ice volume suggested by [Wunsch, 2003]. The model consists of a random walk and ice-volume thresholds. Ice volume increments are random and thus the ice volume itself is a random walk. When the ice volume reaches a certain maximal ice volume $V_{\max }$, it immediately drops to zero; then growth resumes. The ice volume is always positive. Although the ice volume is asymmetric, the low ice volume in glaciation periods is inconsistent with the Vostok ice core and other paleoclimate proxy data since they exhibit long and cold glacial periods. (bottom) Scaling analysis of the model. The ice-volume signal has a scaling behavior (solid circles) as in Vostok ice core with a random-walk exponent up to scale of $100 \mathrm{kyr}$ and a crossover to random behavior for larger scales (not shown). However, the magnitudes of ice-volume increments of the model (open circles) are uncorrelated in contrast to the strong correlations in the Vostok magnitude series. Hence the model fails to reproduce the stochastic nonlinearity we observed in the ice core data.

series $\left|V_{t+1}-V_{t}\right|$ [Ashkenazy et al., 2003a, Figure 1b] while the Wunsch model is a piecewise linear model where the growth itself is linear, the model is not expected to reproduce the magnitude correlations of ice core data. The effect of the maximal ice-volume and zero thresholds is the whitening (i.e., the crossover to "flatter" power spectrum for lower frequencies) of the power spectrum for large scales (low frequencies) since these thresholds restrict the ice volume to be finite (making the time series stationary) and their random appearance leads to a random (white) spectrum. In the bottom panel of Figure 5 we show the root mean square fluctuation function $F(n)$ calculated by the second order detrended fluctuation analysis; while the model's ice volume $V(t)$ is characterized by a scaling exponent which is close to the observed scaling exponent $(\alpha \approx 1.4$ of the model compared to $\alpha=(\beta+1) / 2 \approx 1.6$ of the data; see Figure 2a), the scaling exponent of the magnitude series indicates uncorrelated behavior similar to that of white noise (exponent $\alpha \approx 0.5 \pm 0.05$ compared to $\alpha \approx 0.8$ of the ice core magnitude series; see Figure $3 \mathrm{a}$ ). We thus conclude that the random-walk model plus thresholds is not consistent with the large ice volume during glacial periods, nor with the stochastic nonlinearity of the ice core data as described above (section 2).
[27] We also analyzed the stochastic resonance model for ice-ages of Benzi et al. [1982]. Briefly, this model consists of a two-state system (one cold glacial state and another warm interglacial state) and random transitions between them. This nonlinear model is also forced by a periodic function which enhances the probability of switching from one state to another. We find that the stochastic resonance model does not reproduce the stochastic nonlinearity of the ice core data as described above (section 2). This fact may be understood as follows: Once "stuck" in a state, the fluctuations are as linear as the stochastic forcing while, like in Wunsch model, the sharp temporal nonlinear transitions are not enough to reproduce the intrinsic nonlinearity of the ice core data. It is most probable that the above arguments are also valid for the recent coherence resonance model of Pelletier [2003] which is based on the stochastic resonance model of Benzi et al. [1982].

\section{Models for Glacial Dynamics With Nonlinear Stochastic Forcing}

\subsection{Nonlinear Stochastic Model Based on Nonlinear} External Stochastic Forcing

[28] The stochastic nonlinearity of ice core data as expressed by the magnitude series long range correlations and the broad multifractal spectrum point to an inherently nonlinear stochastic mechanism. Recently, Bacry et al. [2001] proposed a generalized multifractal random-walk model consisting of Gaussian white noise multiplied by a correlated lognormally distributed random variable. The white noise controls the two-point correlations while the correlated noise controls the multifractal spectrum width of the model and hence its nonlinearity. We suggest a model for glacial dynamics based on this multifractal random-walk model. We start with a model for ice-volume dynamics which has self-sustained asymmetric oscillations. Then we add nonlinear noise to account for the stochastic nonlinearity of the ice core data. Ice volume $V$ was observed to be negatively correlated with temperature $T \sim-V$ [Petit et al., 1999], i.e., colder conditions for larger ice volume; thus a model for ice accumulation serves as a model for temperature dynamics.

[29] The assumptions of our model (Figure 6) are as follows.

[30] 1. The ice volume $V$ changes with steps $\delta+b / V$; i.e., $V(t+d t)=V(t)+\delta(t)+b / V(t)$.

[31] 2. When ice volume $V$ "crosses" a critical volume $V_{\max }, b$ is set to be negative, $b=b_{2}<0$. Ice volume is considered to lie between $0.01 V_{\max }$ and $V_{\max }$. When $V=$ $0.01 V_{\max }, b$ becomes $b=b_{1}>0$ until $V$ exceeds the threshold $V_{\max }$.

[32] 3. The ice volume fluctuations $\delta$ are the product of two stochastic inputs, $\delta(t)=\zeta(t) \eta_{i(t)}$. Here $\zeta$ and $\eta$ are Gaussian distributed random variables with zero mean and unit variance.

[33] 4. Random switching between the states $\eta_{i}$ 's is controlled by $i(t)$ which is equal to $[l(t)]$ where [.] stands for the closest integer value. Here $l$ is a random walk described by $l(t+d t)=l(t)+C \omega(t)$, where $C$ is the switching range and $\omega$ is another Gaussian random variable with zero mean and unit variance (see top panel of Figure 6). 


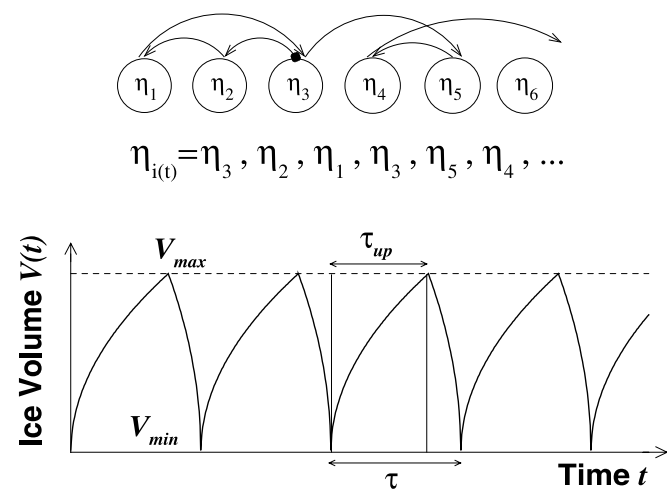

Figure 6. (top) An illustration for the transitions between "oceanic" modes of the thermohaline circulation. We assume that the ocean mode is switched randomly to neighboring modes which are random by themselves. This oceanic noise is coupled to atmospheric noise which is represented by white noise. (bottom) The model's dynamics without noise. The ice volume is periodic and asymmetric with long glacial periods $\left(\tau_{u p}\right)$ followed by short interglacial periods $\left(\tau_{\text {down }}=\tau-\tau_{\text {up }}\right)$ as in the ice core data. When the ice volume reaches a certain maximal ice volume, $V_{\max }$, a negative bias is introduced and the ice volume starts diminishing (melting). When reaching a minimal ice volume, the bias becomes positive and growth begins again.

[34] The first and second assumptions describe the random growth (with $b=b_{1}>0$ ) of the ice sheets and their rapid breakup (with $b=b_{2}<0$ ) after crossing the critical volume $V_{\max }$, similar to the Wunsch model described in section 3.2; such thresholds may be related to the formation of sea ice when the atmospheric temperature drops below a certain temperature or alternatively when the ice sheets are large enough such that the temperature is sufficiently low [c.f. Gildor and Tziperman, 2000; Tziperman and Gildor, 2003] (see also Tziperman lecture, 2001). The $1 / V$ term in the first assumption mimics the reduced ice accumulation for large ice volume, namely the temperature precipitation feedback [Ghil and Treut, 1981; Gildor and Tziperman, 2000] (see also Tziperman lecture, 2001). Ice volume fluctuations, $\delta$, result from two interacting random inputs (third assumption) where one, $\zeta$, may represent the atmosphere, the other, $\eta$, may represent the ocean, and the product, $\zeta \eta$, may represent the atmosphere-ocean interaction. In effect, the random switching that $\eta_{i(t)}$ is based on results in correlated $\eta_{i(t)}$ [Hausdorff et al., 1995; Ashkenazy et al., 2002]. The product $\zeta \eta$ thus forms a product of white noise multiplied by a correlated random variable. Following Bacry et al. [2001], this situation may lead to nonlinearity and multifractality.

[35] The values of $V_{\max }, b_{1}$, and $b_{2}$ are constrained to match the features seen in the natural record (Figure 1). In our simulations we use $d t=0.1 \mathrm{kyr}, V_{\max }=1.5 \sqrt{90 \mathrm{kyr} / d t}$, $b_{1}=1, b_{2}=-3$, and $C=0.27$. We choose the value $90 \mathrm{kyr}$ in $V_{\max }$ so that on average $V$ will grow from zero to $V_{\max }$ after $90 \mathrm{kyr} / d t$ steps. We choose the values of $b_{1}$ and $b_{2}$ such that the ice sheet grows slowly and breaks up rapidly. The switching parameter $C$ determines the number of states $\eta$ for a given number of steps; the number of different states is proportional to the square root of the number of steps (e.g., $\sim 3$ states for $4 \mathrm{kyr}$ and $\sim 5$ for $10 \mathrm{kyr}$ ).

[36] Without noise the model exhibits self-sustained oscillations (Figure 6, bottom panel). The solution of the model without stochastic forcing is periodic,

$$
V(t)=V(\tilde{t}+n \tau)=\left\{\begin{array}{cl}
\sqrt{2 b_{1} \tilde{t}+V_{\min }^{2}} & 0 \leq \tilde{t}<\tau_{u p} \\
\sqrt{2 b_{2}\left(\tilde{t}-\tau_{u p}\right)+V_{\max }^{2}} & \tau_{u p} \leq \tilde{t}<\tau
\end{array},\right.
$$

where $n$ is an integer and

$$
\begin{aligned}
\tau_{u p} & =\frac{V_{\max }^{2}-V_{\min }^{2}}{2 b_{1}}, \\
\tau_{\text {down }} & =\frac{V_{\min }^{2}-V_{\max }^{2}}{2 b_{2}}, \\
\tau & =\tau_{\text {up }}+\tau_{\text {down }}=\frac{1}{2}\left(V_{\max }^{2}-V_{\min }^{2}\right)\left(\frac{1}{b_{1}}-\frac{1}{b_{2}}\right) .
\end{aligned}
$$

Under the current choice of parameters the ice volume is an asymmetric periodic function with long glacial periods followed by short interglacial periods, as seen in the ice core records from both the Arctic and Antarctic.

[37] An example of an arbitrary realization of $400 \mathrm{kyr}$ time series produced by the model with stochastic forcing is shown in Figure 7a. The natural asymmetry of long glacial periods followed by short interglacials is reproduced. The scaling of the model's $V$ series (Figure $7 \mathrm{~b}$ ) indicates random-walk behavior with a power spectrum exponent $\beta \approx 2.2$ (or $\alpha=(\beta+1) / 2=1.6$ ) as seen for the Vostok core, Figure $2 \mathrm{a}$. The magnitude series $|\Delta V|$ is long-range correlated with an $\alpha$ exponent $\sim 0.8$ (Figure $7 \mathrm{c}$ ) as also measured in the climate record of the Vostok core (Figure 3a); the average \pm 1 standard deviation of 10 realizations is $0.8 \pm$ 0.05 . The surrogate data test applied to the $\Delta V$ series changes the magnitude series into an uncorrelated one, indicating the nonlinearity of the model. This nonlinearity is due to the product of the inputs $\eta$ and $\zeta$ in the third assumption. The multifractal spectrum of the time series is broad where, as with the ice core data (Figure $3 \mathrm{~b}$ ), the exponents for negative moments mainly contribute to its broadness (Figure 7d). After the surrogate data test the series becomes linear with a narrow multifractal spectrum and is statistically different from the multifractal spectrum of the original data.

[38] Although the model presented in this subsection reproduces fairly well the statistical properties of the ice core data, it is quite abstract and does not associate specific physical processes with the glacial dynamics. In the next subsection we offer a more realistic stochastic model for climate dynamics.

\subsection{Nonlinear Stochastic Model Based on Nonlinear Interaction Between External Stochastic Linear Forcing and Model's Ice-Volume Variable}

[39] As in the previous subsection, we begin with a model for ice volume that exhibits self-sustained asymmetric oscillations and then add stochastic forcing that mimics the nonlinear stochastic properties of the ice core data. Unlike the previous subsection, the noise added to the 
(a)

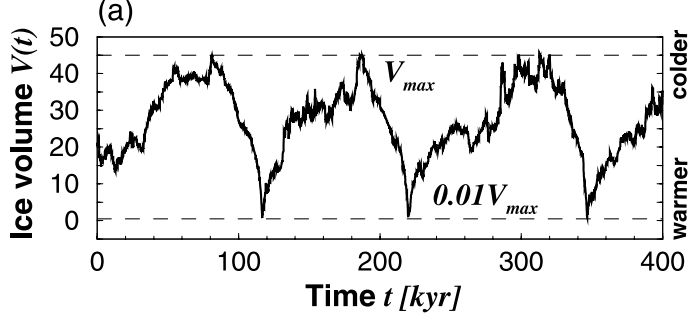

(b)
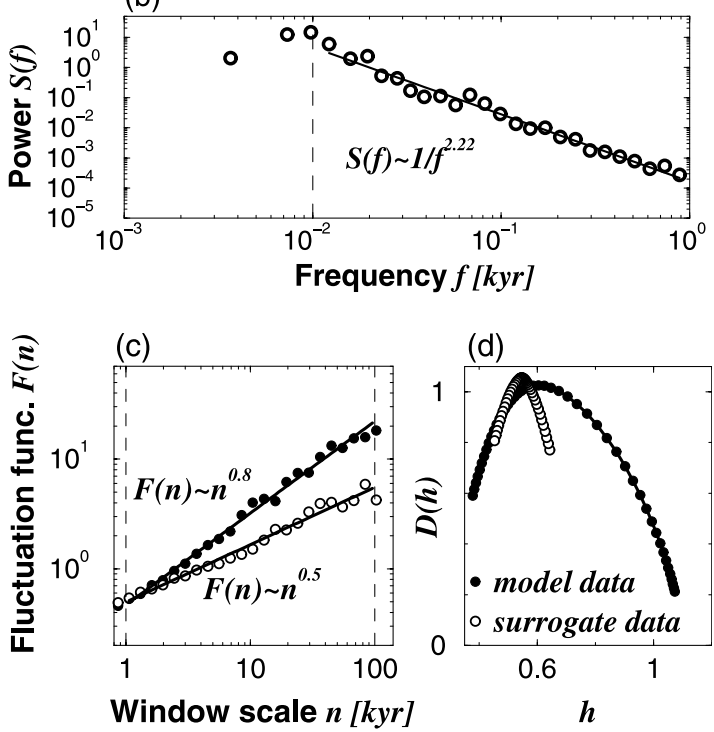

(d)

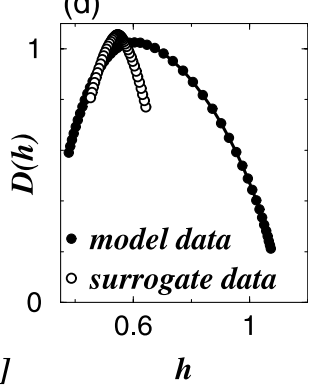

Figure 7. (a) A realization of the nonlinear stochastic model I (section 4.1 and Figure 6) with noise. The asymmetry of the glacial-interglacial oscillations is similar to the asymmetry of the Vostok data. The ice volume is confined to be between the maximum and minimum thresholds (the horizontal dashed lines). (b) The model's power spectrum (of the series shown in Figure 7a) exhibits similar features to that of the Vostok data (Figure 2a). (c, d) Same as Figures 3a and $3 \mathrm{~b}$. The nonlinear properties of the magnitude series power law correlations and the wide multifractal spectrum are reproduced by the model. After applying the surrogate data test for nonlinearity (Appendix B), the magnitude series becomes uncorrelated and the multifractal spectrum becomes much narrower as also seen in the Vostok data.

system is not independent of the model's dynamics, but rather interacts with the model's ice-volume variable.

[40] The deterministic model presented here is a simplified version of the sea-ice-switch model of Gildor and Tziperman [2001] [see Ashkenazy and Tziperman, 2004]. This simplification allows easier construction of a nonlinear stochastic model that accounts for the nonlinear features found in the Vostok and other ice cores. The model's assumptions are as follows.

[41] 1. Changes in ice volume $V(t)$ equal net precipitation $P$ (snow over land) minus ablation $S$ (the sum of all processes that reduce the ice sheet volume including melting, wind erosion, glacial surges, etc.). We assume that the precipitation is large for small ice volume and small for large ice volume, namely the temperature-precipitation feedback [Ghil and Treut, 1981; Gildor, 2003]. We also assume that the ablation rate is constant. Changes in ice volume may be written as

$$
\frac{d V}{d t}=P-S=\left(p_{0}-k V\right)-S,
$$

where $p_{0}$ is the precipitation rate in the absence of ice and $k$ is the ice-volume growth rate constant.

[42] 2. When the ice volume crosses a maximal ice volume $V_{\max }$ the precipitation rate reduces by a factor $1-a_{o n}$ where $0<a_{\text {on }}<1 ; a_{\text {on }}$ represents the relative sea-ice area (sea-ice area versus ocean area) when it exists. When the ice volume is reduced below a minimal ice volume, $V_{\min }$, precipitation returns to the normal rate. This assumption reflects the seaice-switch mechanism [Gildor and Tziperman, 2001]; when the ice volume is sufficiently large, the ocean temperature drops (due to the increased albedo) below the freezing temperature of seawater and a large sea-ice cover forms. This leads to reduced precipitation over land due to: (1) less evaporation due to the insulation effect of the sea ice, (2) colder conditions caused by the high albedo of the sea ice, and (3) the shift of the storm track toward the equator [Gildor and Tziperman, 2000]. The model's dynamics are thus described by

$$
\frac{d V}{d t}=\left(p_{0}-k V\right)(1-a)-S,
$$

where $a=0$ when the sea-ice switch is OFF and $a=a_{o n}(0<$ $\left.a_{o n}<1\right)$ when the sea-ice switch is ON.

[43] 3. The ice volume is subject to random fluctuations, $\delta(t)$

$$
\delta(t)=\zeta(t)\left[A_{1} O(t)+A_{2}\right],
$$

where $\zeta(t)$ is Gaussian white noise, $A_{1}$ and $A_{2}$ are constant parameters, and $O(t)$ is

$$
O(t)=\int_{t-T}^{t} \frac{d V}{d t^{\prime}} d t^{\prime}=V(t)-V(t-T),
$$

where $T$ is constant. We consider $\zeta(t)$ to represent rapid atmospheric fluctuations [Hasselmann, 1976] and $O(t)$ to represent slow oceanic fluctuations. We assume that climate fluctuations result partly from atmosphere-ocean interaction ( $A_{1}$ parameter) and partly from fast atmospheric fluctuations ( $A_{2}$ parameter). The oceanic fluctuations, $O(t)$, are assumed to be the sum of changes in ice volume; surges and melted ice are assumed to change ocean temperature. The response time $T$ in equation (2) is assumed to be the mixing time of the ocean. The model's final dynamical equation is then

$$
\frac{d V}{d t}=\left(p_{0}-k V\right)(1-a)-S+\zeta(t)\left\{A_{1}[V(t)-V(t-T)]+A_{2}\right\} .
$$

[44] In our simulation we used the following parameter values: $p_{0}=0.25 \mathrm{~Sv}\left(1 \mathrm{~Sv} \equiv 10^{6} \mathrm{~m}^{3} / \mathrm{s}\right), S=0.21 \mathrm{~Sv}, k=$ $1 /(40 \mathrm{kyr}), a_{o n}=0.3, A_{1}=0.29 \mathrm{kyr}^{-1}, A_{2}=0.062 \mathrm{~Sv}, T=$ $2.3 \mathrm{kyr}$, and $d t=0.1 \mathrm{kyr}$. The values of $p_{0}, a_{o n}$, and $S$ are consistent with previous studies [Gildor and Tziperman, 2000; Tziperman and Gildor, 2003; Ashkenazy and Tziperman, 2004], $k$ is chosen according to Imbrie and Imbrie 


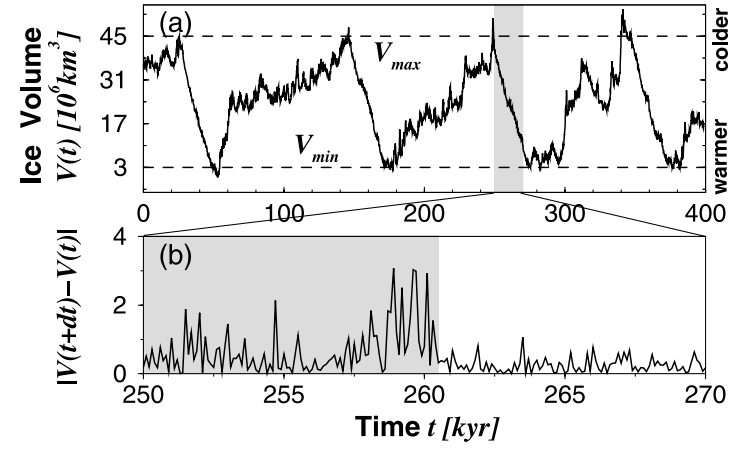

Figure 8. (a) A realization of the nonlinear stochastic model II (section 4.2). As in Figure 7a, the asymmetry of the glacial-interglacial oscillations is similar to that in the Vostok data. The ice volume is confined to be between minimum and maximum thresholds (the horizontal dashed lines). (b) An enlargement from Figure 8a showing the clustering of magnitudes of ice-volume increment, similar to what we observed in the data $\delta D$ from the Vostok ice core [see Ashkenazy et al., 2003a]. The shaded area shows a region of pronounced fluctuations.

[1980] and Pelletier [1997], $A_{1}$ and $A_{2}$ are tuned to reproduce the stochastic nonlinearity of the data, and $T$ is on the order of the mixing time of the ocean [Broecker, 1991; Hartmann, 1994] (i.e., few thousands of years).

[45] Without the stochastic fluctuations, the model exhibits self-sustained asymmetric oscillations, similar to that of Gildor and Tziperman [2001] and Tziperman and Gildor [2003]; i.e., long glacial periods followed by short interglacial periods. When $A_{1}$ is set to be zero, the model equation accumulates white noise $A_{2} \zeta(t)$ on top of the intrinsic oscillations of the model. The random fluctuations will cause changes in the fixed period of the glacial oscillations but will not change the asymmetry of the model's dynamics. The fast atmospheric fluctuations represented by $A_{2} \zeta(t)$ will not cause changes in the stochastic nonlinear properties of the climate records as described above. The nonlinear term, $A_{1} \zeta(t) O(t)$, which represents the atmosphere-ocean interaction, controls the nonlinearity of the model's ice volume.

[46] One realization of the model's ice volume is shown in Figure 8. The asymmetry of the ice-volume record is reproduced by the model (Figure 8a) and also the clustering of magnitudes of ice-volume increments (Figure 8b). The two-point correlation function (the root mean square fluctuation function) of the model's ice volume is shown in Figure $9 \mathrm{a}$ and exhibits long-range correlations, as seen in the Vostok ice core data; the exponent $\alpha \approx 1.6$ (Figure 9a) is equivalent to the power spectrum exponent $\beta=2.2$ of the natural data (Figure 2a) since $\beta=2 \alpha-1$. As expected, the two-point correlations remain unchanged after the Fourier phase randomization test (Figure 9a). The magnitude series $|V(t+d t)-V(t)|(d t=0.1 \mathrm{kyr})$ is correlated with exponent $\alpha \approx 0.8$ (Figure $9 \mathrm{~b}$, the average \pm standard deviation of 10 realizations is $0.8 \pm 0.05$ ) as seen in the Vostok ice core data (Figure 3a); the magnitude series obtained from the surrogate (linearized) phased randomized series is uncorrelated with exponent $\alpha \approx 0.5 \pm 0.05$ where this exponent is significantly different from the exponent of the original model's ice-volume series (presented in this subsection).
This test confirms that indeed our model exhibits stochastic nonlinear behavior. The multifractal spectrum of the model's ice volume is broad and becomes significantly narrower for the surrogate (linearized) ice-volume series (Figures 9c and 9d). This multifractal spectrum is also similar to the multifractal spectrum of the natural data (Figure 3b).

[47] Unlike the model presented in section 4.1, the fluctuations $\delta(t)$ depend on the model's variable, $V(t)$ (equations (1) and (2)). It follows from equation (1) that when changes in ice volume are small, the nonlinearity decreases. When the mixing time $T$ is increased, the term $V(t)-V(t-T)$ is in general larger and thus the nonlinearity is expected to increase (Figure 10a). In addition, when the amplitude of the oceanic noise $A_{1}$ increases, the stochastic nonlinearity of the model strengthens (Figure 10b). However, the model's stochastic nonlinearity is almost insensitive to small changes $(\sim 10 \%)$ in these two parameters.

[48] In general, other deterministic climate models may be generalized to reproduce the nonlinear stochastic characteristics of paleoclimate data. This can be done by coupling one of the model's variables to external noise. It should be noted, however, that such an approach will not

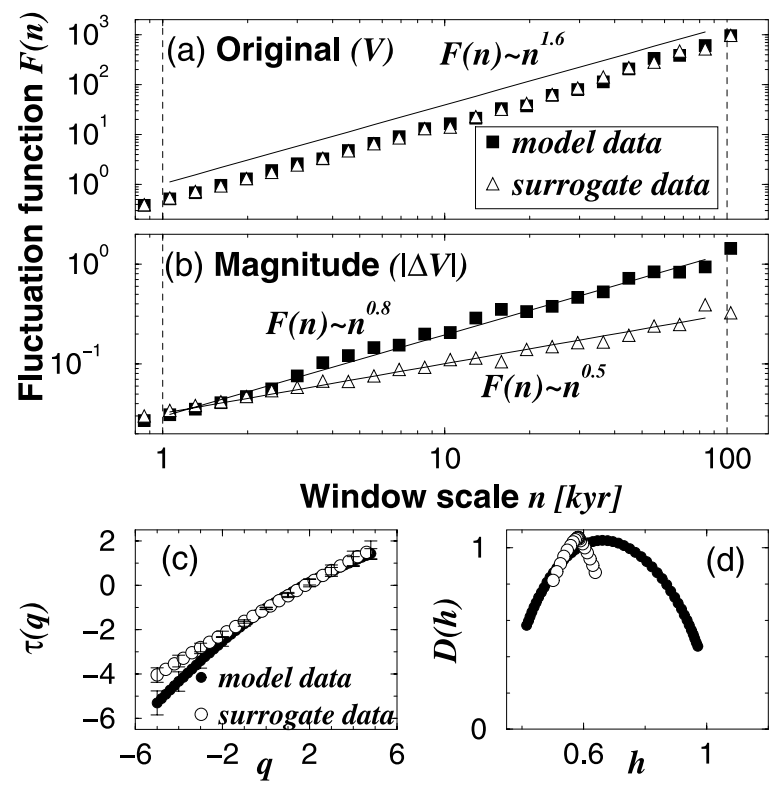

Figure 9. The root mean square fluctuation function $F(n)$ calculated using second-order detrended fluctuation analysis (see Appendix C) of (a) the model ice volume $V(t)$ shown in Figure 8 and (b) the magnitudes of ice-volume increments $|V(t+d t)-V(t)|$ before (squares) and after (triangles) the phase randomization test (Appendix B). (c) The exponents $\tau(q)$ of different moments $q$ before (solid circles) and after (open circles) the phase randomization test. (d) The multifractal spectrum $D(h)$ before (solid circles) and after (open circles) the phase randomization procedure. Note that the magnitude series is correlated and the multifractal spectrum is wide before the phase randomization procedure and the magnitude series is uncorrelated and the multifractal spectrum is narrow after the phase randomization procedure, indicating the stochastic nonlinearity of the model. These results are similar to what we observed in the Vostok data (Figure 3 and Ashkenazy et al. [2003a]). 

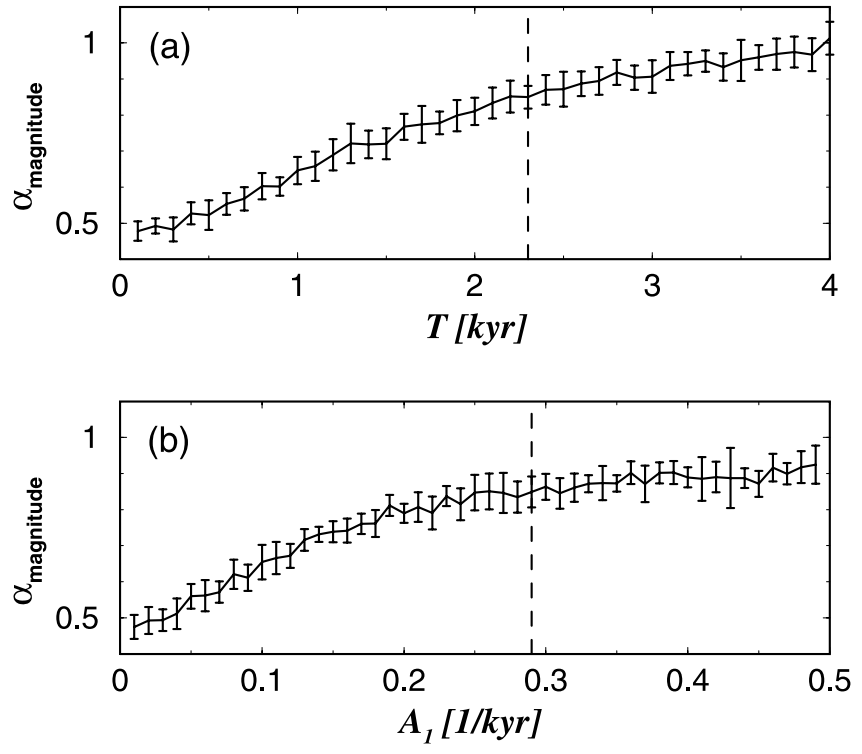

Figure 10. (a) Sensitivity test for the nonlinearity of the nonlinear stochastic model II (section 4.2). Here we change the parameter representing the mixing time of the ocean $T$ and estimate the nonlinear measure of correlation exponent of the magnitudes of ice-volume increments (mean of 10 realizations \pm 1 standard deviation); the other model's parameters are as in Figures 8 and 9. The vertical dashed line indicates the value used in Figures 8 and 9. It is apparent that the model's nonlinearity is insensitive to small changes $(\sim 10 \%)$ in the mixing time $T$. (b) Same as Figure 10a for the amplitude of the oceanic noise $A_{1}$. Also here the model is robust to small changes in the amplitude of the oceanic noise $A_{1}$. The vertical dashed lines indicate the values used in Figures 8 and 9.

necessarily lead to a reasonable reconstruction of both the magnitude series correlations as well as the multifractal spectrum; the nonlinear dynamics of climate models make this reconstruction difficult. It took us considerable effort to construct the stochastic nonlinear climate models presented in this paper, and many were rejected before suitable ones were found.

\section{Summary}

[49] Paleoclimate proxy records show evidence for both Milankovitch forcing and stochastic variability. The Milankovitch forcing is usually attributed to the presence of Milankovitch frequencies in climate proxy records, although the process is most probably nonlinear. On the other hand, the power spectra of the proxy records also show red-noise behavior, which may be a sign of a stochastic underlying process. The majority of the power spectrum is governed by the non-Milankovitch frequencies. Thus we assume that the underlying process is mainly driven by stochastic elements.

[50] Climate change fluctuations are in some cases considered to be due to linear stochastic noise. Recently, it has been shown that this is not the case and that the noise fluctuations have a unique pattern of clustering into more and less volatile regions of climate fluctuations [Ashkenazy et al., 2003a]. This pattern is reflected by correlations of the magnitudes of climate increments and in a broad multifractal spectrum; this pattern is not necessarily related to the large-scale glacial dynamics. Here we propose two mechanisms that may lead to such statistical properties. These mechanisms involve interaction between a slowly varying process (like the ocean) with a rapidly varying process (like the atmosphere). The slow process might be external or related to the internal dynamics of the model. This approach is in some sense different than the approach of Hasselmann [1976] where he proposed that on short timescales one sees the rapidly varying atmospheric fluctuations while on long timescales the oceanic fluctuations (which result from accumulation of the fast atmospheric processes) are dominant. Our results and models suggest that in fact even on large timescales, rapid atmospheric fluctuations may amplify the slow oceanic fluctuations.

[51] The stochastic nonlinearity of ice core data may be used to test (1) stochastic models, (2) realistic models (like general circulation ocean-atmosphere models), and (3) simple deterministic models that reproduce the red-noise spectrum of paleoclimate data. It is not guaranteed that addition of external nonlinear noise to a deterministic model will produce the correct stochastic nonlinearity, because glacial models usually contain complex, nonlinear dynamics.

[52] Many models for glacial dynamics are deterministic models that are forced by insolation changes. These kinds of models are, in most cases, insufficient to account for the red-noise spectrum of the climatic records. Moreover, even if they account for the red-noise spectrum, it is unlikely that they would reproduce the stochastic nonlinear properties found in the ice core records. Instead, realistic models should include both deterministic and stochastic elements. Our approach was to first derive the general pattern of the climate records (long glacial period followed by short interglacial period) and then to add the noise to the system. In order to reproduce the stochastic nonlinear properties of the data, it is necessary that the noise be nonlinear (either due to nonlinear external noise or due to linear noise that interacts with the system's variables). It is unlikely that nonlinear recording underlies the stochastic nonlinear properties of paleoclimate data since these properties point to unique power laws and a unique multifractal spectrum suggesting processes that are time invariant and involve "interaction" between the slow and fast modes of the system. Moreover, a monotonic nonlinear transformation, as nonlinear recording is expected to be, is not considered to be linear according to the definition we use [Schreiber and Schmitz, 2000], and after applying the surrogate test for nonlinearity of Schreiber and Schmitz [2000] the paleoclimate data is clearly nonlinear. While we took Gildor and Tziperman's [2000] as the base deterministic model, our approach can be applied to other climate models, which ideally should reproduce the nonlinear stochastic features of the climate records. We proposed two models that reproduce the nonlinear statistical characteristics of ice core proxy records. We describe the nonlinear properties of the paleoclimate proxy records as an interaction between slow oceanic processes and fast atmospheric processes. We recognize that it is still necessary to identify in a more accurate way the processes that underlie the nonlinear stochastic properties of the ice core data. However, to our 
knowledge, there are no physically motivated models that explain the stochastic nonlinear properties described here. Thus the models suggested here are an important step toward a more complete understanding of Earth's climate system.

\section{Appendix A: Nonlinearity and Multifractality}

[53] In this appendix we define linearity and nonlinearity of a time series. We also review the relation between the multifractality of a time series and its nonlinearity.

[54] Following Theiler et al. [1992] and Schreiber and Schmitz [1996, 2000], we define a time series to be "linear" if it is possible to reproduce its statistical properties from the power spectrum and the probability distribution alone, regardless of the Fourier phases
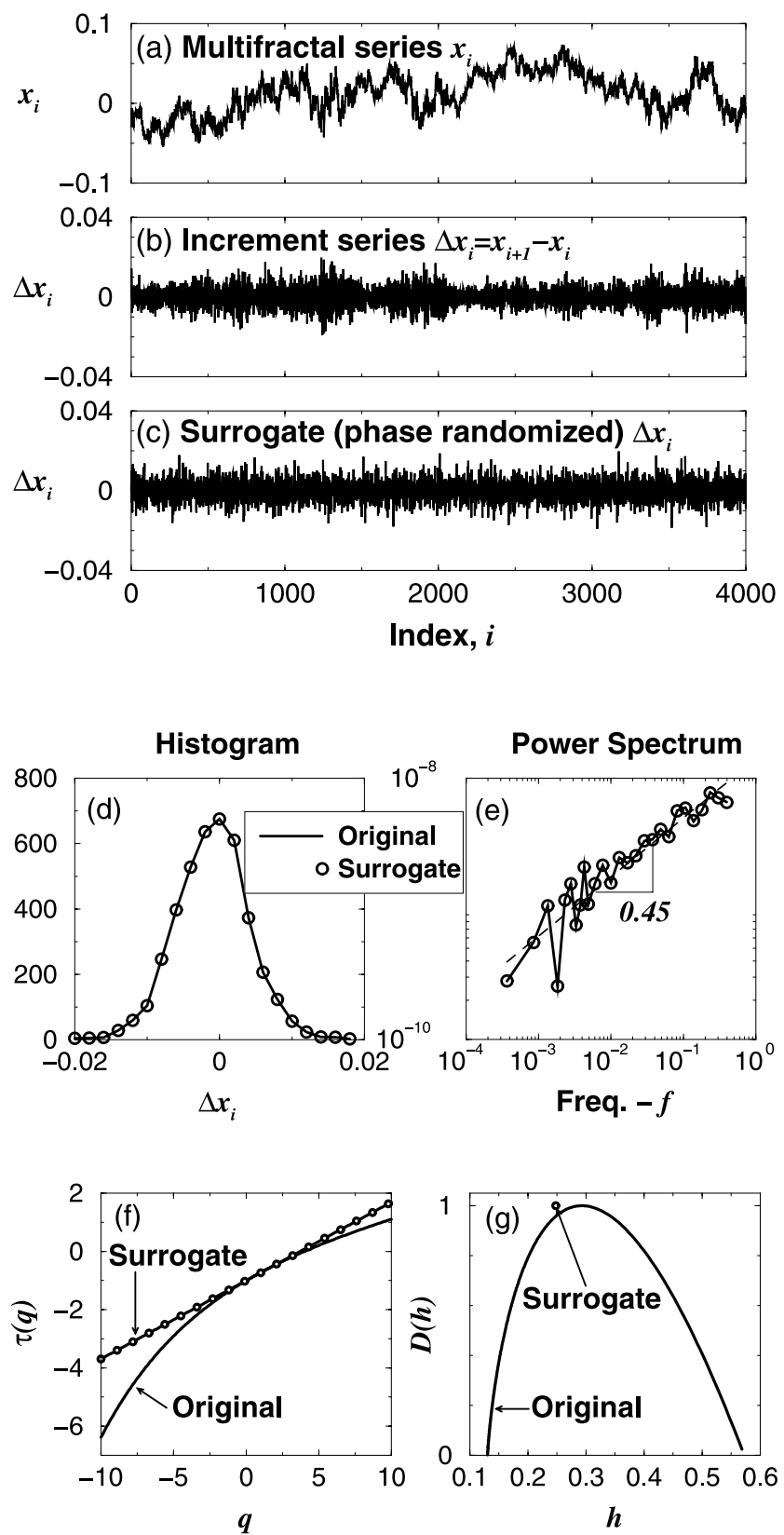

[Schreiber and Schmitz, 2000]. This definition includes (1) autoregression processes,

$$
x_{n}=\sum_{i=1}^{M} a_{i} x_{n-i}+\sum_{i=0}^{L} b_{i} \eta_{n-i},
$$

where $\eta$ is Gaussian white noise, and (2) fractional Brownian motion [Mandelbrot and van Ness, 1968]; the output, $x_{n}$, of these processes may undergo monotonic nonlinear transformations,

$$
s_{n}=s\left(x_{n}\right)
$$

and still be linear [Schreiber and Schmitz, 2000]. Thus the probability distribution of $s_{n}$ does not influence the nonlinearity as defined here. Processes which are not linear are defined as "nonlinear." It is possible to destroy the nonlinearity of a time series by randomizing its Fourier phases [see Schreiber and Schmitz, 2000, 1996]; see Appendix B.

Figure A1. (a) A nonlinear (multifractal) time series generated according to Arneodo et al. [1998] log-Poisson algorithm. (b) The increment series of Figure A1a. The "clustering" into small and big fluctuations reflects the stochastic nonlinearity of the multifractal time series. (c) The surrogate (phase randomized data) series of the increment series (Figure A1b). To generate the surrogate data, we use the procedure of Schreiber and Schmitz [2000] that keeps the histogram and the power spectrum of the original time series almost unaffected but randomizes the Fourier phases. The "clustering" of the original time series (Figure A1b) is not visible any more after randomizing the Fourier phases, indicating that indeed phase randomization procedure destroyed the nonlinearity of the original increment time series (Figure A1b). (d) The histogram of the increment series of the original increment series (Figure A1b) and of the surrogate increment series from Figure A1c. As expected, the histogram of the original increment series (solid line) is identical to the histogram of the surrogate data (open circles). (e) The power spectra of the original increment data shown in Figure A1b and of the surrogate data shown in Figure A1c. As expected, the power spectra are identical to each other, both indicating anticorrelation with a correlation exponent $\beta=-0.45$ ( $\beta$ is approximated by regression of $S(f) \sim 1 / f^{\beta}$ to the power spectrum). In Figures A1d and A1e, we demonstrate that although the histogram and power spectrum of the original increment data is maintained, the surrogate data shown in Figure A1c are very different from the original data shown in Figure A1b. (f) The analytical scaling exponents of different moments $\tau(q)$ [Arneodo et al., 1998] for the original series shown in Figure A1a and for the integrated surrogate series shown in Figure A1c. The $\tau(q)$ is curved for the original nonlinear multifractal series and is straight for the linearized time series. (g) The analytical multifractal spectrum $D(h)$ [Arneodo et al., 1998] for the original series shown in Figure A1a and for the integrated surrogate series shown in Figure A1c. The multifractal spectrum is wide for the nonlinear multifractal series, and it collapses to a point for the linearized surrogate time series. 
[55] The nonlinearity (or linearity) of a time series is related to its multifractality. The definition of multifractality is based on the partition function $Z_{q}(l)$ of a time series, $s_{n}$, and may be defined as [Barabasi and Vicsek, 1991]

$$
Z_{q}(l)=\left\langle\left|s_{n+l}-s_{n}\right|^{q}\right\rangle,
$$

where \langle\rangle stands for expectation value. In some cases, $Z_{q}(l)$ obeys scaling laws,

$$
Z_{q}(l) \sim l^{\zeta_{q}}
$$

If the exponents $\zeta_{q}$ are linearly dependent on $q$, the series $s_{n}$ is "monofractal"; otherwise $s_{n}$ is "multifractal" [e.g., Muzy et al., 1994; Bacry et al., 2001]. Note that in the present study we use an advanced method, the wavelet transform modulus maxima method, to accurately estimate the exponents of negative moments $q<0$ (for details, see Muzy et al. [1994]). This method uses a wavelet transform that eliminates polynomial trends from the data; in our analysis we used the eight-tap Daubechies discrete wavelet transform [Daubechies, 1992].

[56] The two-point correlation function of an increment time series $\Delta s_{n} \equiv s_{n+1}-s_{n}$ is defined as

$$
A(l)=\left\langle\Delta s_{n} \Delta s_{n+l}\right\rangle .
$$

For long-range correlated stationary Gaussian time series,

$$
A(l) \sim l^{-\gamma},
$$

where $0<\gamma<1$. In this case the exponent $\gamma$ is related to the detrended fluctuation analysis exponent $\alpha$ of $\Delta s_{n}$ series by [Taqqu et al., 1995; Makse et al., 1996]

$$
Z_{2}(l) \sim\left\langle s_{n} S_{n+l}\right\rangle \sim l^{\zeta_{2}} \sim l^{2-\gamma}=l^{2 \alpha} .
$$

In addition, these exponents are related to the power spectrum exponent $\beta$ of the increment series $\Delta s_{n}$,

$$
S(f) \sim 1 / f^{\beta},
$$

by

$$
\beta=1-\gamma=2 \alpha-1
$$

Thus the second moment only depends on the power spectrum and is independent of the Fourier phases,

$$
\zeta_{2}=2 \alpha=\beta+1 .
$$

[57] For monofractal series [Muzy et al., 1993, 1994],

$$
\zeta_{q}=\alpha q=\frac{\beta+1}{2} q .
$$

Thus the multifractal spectrum of a monofractal series is independent of the Fourier phases. This implies that (1) Gaussian long-range correlated time series with uncorrelated Fourier phases are monofractal and (2) after randomizing the Fourier phases of a multifractal series, it becomes monofractal.

[58] In summary, monofractal series are linear since their statistical properties depend only on the power spectrum (two-point correlations) and the probability distribution. On the other hand, multifractal series are nonlinear since their higher moments are not solely dependent on the probability distribution and the power spectrum but are also related to the Fourier phases; see Figure A1.

\section{Appendix B: Surrogate Test for Nonlinearity}

[59] Schreiber and Schmitz [1996, 2000] suggested an algorithm which preserves both the original probability distribution of the time series and its Fourier amplitudes but randomizes the Fourier phases; thus this algorithm preserves the linear properties of the time series but destroys the nonlinear properties stored in the Fourier phases. The algorithm is iterative and consists of the following steps: (1) Store a sorted list of the original data $\left\{h_{k}\right\}$ and the power spectrum $\left\{S_{n}\right\}$ of $\left\{h_{k}\right\}$. (2) Begin $(l=0)$ with a random shuffle $\left\{h_{k}^{(l=0)}\right\}$ of the data $\left\{h_{k}\right\}$. (3) Replace the power spectrum $\left\{S_{n}^{(l)}\right\}$ of $\left\{h_{k}^{(l)}\right\}$ by $\left\{S_{n}\right\}$ (keeping the Fourier phases of $\left\{S_{k}^{(l)}\right\}$ ) and then transform back. (4) Sort the series obtained from step 3. (5) Replace the sorted series from step 4 by the sorted $\left\{h_{k}\right\}$ and then return to the presorting order (i.e., the order of the series obtained from step 3); the resulting series is $\left\{h_{k}^{(l+1)}\right\}$. Repeat steps $3-5$ until convergence, i.e., until series from consecutive iterations will be almost the same.

[60] In order to check if a time series is linear or nonlinear, it is possible to generate many surrogate linear time series out of the original time series and then to check how significant the nonlinearity of the original time series is compared to the linearized surrogate time series; see Figure A1. There are some limitations of the Schreiber and Schmitz [2000] surrogate data test for nonlinearity: The different realizations may not be completely independent of each other and thus not uniformly span the probability space [see Dolan and Spano, 2001]. We also applied more advanced techniques that handle this technical problem and obtained similar results.

\section{Appendix C: Detrended Fluctuation Analysis (DFA)}

[61] Natural time series often exhibit irregular and complex behavior. In many cases the time series are masked by constant, linear, and polynomial trends. These trends are not necessarily related to the intrinsic dynamics of the time series and may cause erroneous estimation of the scaling exponents; trends are also known to affect the power spectrum. Peng et al. [1994] proposed a simple method, the detrended fluctuation analysis (DFA), to eliminate constant trends from natural time series; later, this technique was generalized to exclude also higher-order polynomial trends [Bunde et al., 2000].

[62] The DFA consists of the following steps:

[63] 1. Given a time series $x_{i}$, create the profile,

$$
y(k)=\sum_{i=1}^{N}\left(x_{i}-\left\langle x_{i}\right\rangle\right),
$$

where $\langle\cdot\rangle$ stands for the average and $N$ is the number of elements in the time series.

[64] 2. Divide $y(k)$ into windows of size $n$. For each window calculate the local polynomial trend $y_{n}(k)$ by least square fitting. 
[65] 3. Calculate the root mean square fluctuation function $F(n)$ by subtracting from the profile $y(k)$ the local polynomial trend $y_{n}(k)$,

$$
F(n) \equiv \sqrt{\frac{1}{N} \sum_{k=1}^{N}\left[y(k)-y_{n}(k)\right]^{2}}
$$

[66] 4. Calculate $F(n)$ for different window size; when the series follows scaling laws, then $F(n) \sim n^{\alpha}$. This exponent can be estimated from the slope in a double logarithmic (log-log) plot.

[67] The exponent $\alpha$ is related to the power spectrum exponent $\beta\left(S(f) \sim 1 / f^{\beta}\right)$ by $\beta=2 \alpha-1$. The $m$-order DFA eliminates polynomial trends of order $m-1$ from the data. When $\alpha=0.5$, the series is uncorrelated and corresponds to white noise with $\beta=0$. When $\alpha>0.5$, the series is correlated and values in the series tend to persist; this is because the low frequencies prevail $(\beta>0)$. When $\alpha<0.5$, the series is anti-correlated and tends to alternate since the high frequencies prevail $(\beta<0)$.

[68] Acknowledgments. We thank S. Havlin for helpful discussions. H.G. is supported by the Clore Foundation. Y.A. is supported by the Yeshaya Horowitz Association through Center for Complexity Science.

\section{References}

Arneodo, A., E. Bacry, and J. F. Muzy (1998), Random cascades on wavelet dyadic trees, J. Math. Phys., 39, 4142-4164.

Ashkenazy, Y., and E. Tziperman (2004), Are the 41 kyr glacial oscillations a linear response to Milankovitch forcing?, Quat. Sci. Rev., 23, 18791890 .

Ashkenazy, Y., P. C. H. Ivanov, S. Havlin, C.-K. Peng, A. L. Goldberger, and H. E. Stanley (2001), Magnitude and sign correlations in heartbeat fluctuations, Phys. Rev. Lett., 86, 1900-1903.

Ashkenazy, Y., J. M. Hausdorff, P. C. H. Ivanov, and H. E. Stanley (2002), A stochastic model of human gait dynamics, Physica A, 316, $662-670$.

Ashkenazy, Y., D. R. Baker, H. Gildor, and S. Havlin (2003a), Nonlinearity and multifractality of climate change in the past 420,000 years, Geophys. Res. Lett., 30(22), 2146, doi:10.1029/2003GL018099.

Ashkenazy, Y., S. Havlin, P. C. h. Ivanov, C.-K. Peng, V. Schulte-Frohlinde, and H. E. Stanley (2003b), Magnitude and sign scaling in power-law correlated time series, Physica A, 323, 19-41.

Bacry, E., J. Delour, and J. Muzy (2001), Multifractal random walk, Phys. Rev. E, 64, 026103-026106.

Barabasi, A. L., and T. Vicsek (1991), Multifractality of self-affine fractals, Phys. Rev. A, 44, 2730-2733.

Benzi, R., G. Parisi, A. Sutera, and A. Vulpiani (1982), Stochastic resonance in climatic change, Tellus, 34, 10-16.

Berger, A., and M. F. Loutre (1991), Insolation values for the climate of the last 10 million years, Ouat. Sci. Rev., 10, 297-317.

Berger, A., and M. F. Loutre (1996), Modeling the climate response to astronomical and $\mathrm{CO}_{2}$ forcing, C. R. Acad. Sci., Ser. IIa Sci Terre Planetes, 323, 1-16.

Broecker, W. S. (1991), The great ocean conveyor, Oceanography, 4, 7989.

Bunde, A., and S. Havlin (Eds.) (1996), Fractals in Science, 2nd ed., Springer, New York.

Bunde, A., S. Havlin, J. W. Kantelhardt, T. Penzel, J. H. Peter, and K. Voigt (2000), Correlated and uncorrelated regions in heart-rate fluctuations during sleep, Phys. Rev. Lett., 85, 3736-3739.

Daubechies, I. (1992), Ten Lectures on Wavelets, Soc. for Indust. and Appl. Math., Philadelphia, Pa.

Dolan, K. T., and M. L. Spano (2001), Surrogate for nonlinear time series analysis, Phys. Rev. E, 64, 046128

Ghil, M., and H. L. Treut (1981), A climate model with cryodynamics and geodynamics, J. Geophys. Res., 86, 5262-5270.

Gildor, H. (2003), When Earth's freezer door is left ajar, Eos Trans. $A G U$, 84(23), 215.
Gildor, H., and E. Tziperman (2000), Sea ice as the glacial cycles climate switch: role of seasonal and orbital forcing, Paleoceanography, 15, 605615 .

Gildor, H., and E. Tziperman (2001), A sea-ice climate-switch mechanism for the 100-kyr glacial cycles, J. Geophys. Res., 106, 9117-9133.

Hagelberg, T., N. Pisias, and S. Elgar (1991), Linear and nonlinear couplings between orbital forcing and the marine $\delta^{18} O$ record during the late Neogene, Paleoceanography, 6, 729-746.

Hagelberg, T. K., G. Bond, and P. Demenocal (1994), Milankovitch band forcing of sub-Milankovitch climate variability during the Pleistocene, Paleoceanography, 9, 545-558.

Hartmann, D. (1994), Global Physical Climatology, Elsevier, New York.

Hasselmann, K. (1976), Stochastic climate models: I. Theory, Tellus, 28, $473-485$.

Hausdorff, J. M., C.-K. Peng, Z. Ladin, J. Y. Wei, and A. L. Goldberger (1995), Is walking a random walk? Evidence for long-range correlations in the stride interval of human gait, J. Appl. Physiol., 78, 349-358.

Huybers, P., and C. Wunsch (2004), A depth-derived Pleistocene age model: Uncertainty estimates, sedimentation variability, and nonlinear climate change, Paleoceanography, 19, PA1028, doi:10.1029/2002PA000857.

Imbrie, J., and J. Z. Imbrie (1980), Modelling the climatic response to orbital variations, Science, 207, 943-953.

Imbrie, J., J. Hays, D. Martinson, A. McIntyre, A. Mix, J. Morley, N. Pisias, W. Prell, and N. Shackleton (1984), The orbital theory of Pleistocene climate: Support from a revised chronology of the marine $\delta^{18} \mathrm{O}$ record, in Milankovitch and Climate: Part I, pp. 269-305, Springer, New York.

Imbrie, J., et al. (1992), On the structure and origin of major glaciation cycles: 1. Linear responses to Milankovitch forcing, Paleoceanography, 7, 701-738.

Imbrie, J., et al. (1993), On the structure and origin of major glaciation cycles: 2. The 100,000-year cycle, Paleoceanography, 8, 699-735.

King, T. (1996), Quantifying nonlinearity and geometry in time series of climate, Quat. Sci. Rev., 15, 247-266.

Kominz, M., and N. Pisias (1979), Pleistocene climate-Deterministic or stochastic, Science, 204, 171-173.

Lovejoy, S., and D. Schertzer (1986), Scale invariance of climatological temperatures and the spectral plateau, Ann. Geophys., Ser. B, 4, 401409 .

Makse, H. A., S. Havlin, M. Schwartz, and H. E. Stanley (1996), Method for generating long-range correlations for large systems, Phys. Rev. E, 53, 5445-5449.

Mandelbrot, B. B., and J. W. van Ness (1968), Fractional brownian motions, fractional noises and applications, SIAM Rev., 10, 422-437.

Milankovitch, M. (1941), Canon of insolation and the ice-age problem (in German), Spec. Publ. 132, R. Serb. Acad., Belgrade. (English translation, Isr. Program for Sci. Transl., Jerusalem, 1969.)

Muzy, J., E. Bacry, and A. Arneodo (1993), Multifractal formalism for fractal signals: The structure function approach versus the wavelettransform modulus-maxima method, Phys. Rev. E, 47, 875-884.

Muzy, J., E. Bacry, and A. Arneodo (1994), The multifractal formalism revisited with wavelets, Int. J. Bifurcat. Chaos, 4, 245-302.

Neeman, B. (1993), Orbital tuning of paleoclimate records: A reassessment, Tech. Rep. LBNL-39572, Lawrence Berkeley Natl. Lab., Berkeley, Calif.

Paillard, D. (1998), The timing of Pleistocene glaciations from a simple multiple-state climate model, Nature, 391, 378-381.

Pelletier, J. D. (1997), Analysis and modeling of the natural variability of climate, J. Clim., 10, 1331-1342.

Pelletier, J. D. (2003), Coherence resonance and ice ages, J. Geophys. Res., 108(D20), 4645, doi:10.1029/2002JD003120.

Peng, C.-K., S. Buldyrev, S. Havlin, M. Simons, H. E. Stanley, and A. L. Goldberger (1994), Mosaic organization of DNA nucleotides, Phys. Rev. E, 49, 1685-1689.

Petit, J. R., et al. (1999), Climate and atmospheric history of the past 420,000 years from the Vostok ice core, Antarctica, Nature, 399, 429436.

Raymo, M. E. (1997), The timing of major terminations, Paleoceanography, 12, 577-585.

Roe, G. H., and M. Allen (1999), A comparison of competing explanations for the 100,000-yr ice age cycle, Geophys. Res. Lett., 26, 2259-2262.

Saltzman, B. (1990), Three basic problems of paleoclimatic modeling: A personal perspective and review, Clim. Dyn., 5, 67-78.

Saltzman, B., and A. Sutera (1987), The mid-Quaternary climate transition as the free response of a three-variable dynamical model, J. Atmos. Sci., $44,236-241$

Schmitt, F., S. Lovejoy, and D. Schertzer (1995), Multifractal analysis of the Greenland ice-core project climate data, Geophys. Res. Lett., 22, $1689-1692$

Schreiber, T., and A. Schmitz (1996), Improved surrogate data for nonlinearity tests, Phys. Rev. Lett., 77, 635-638. 
Schreiber, T., and A. Schmitz (2000), Surrogate time series, Physica D, 142, $346-382$.

Shlesinger, M. F. (1987), Fractal time and $1 / f$ noise in complex systems, Ann. N. Y. Acad. Sci., 504, 214-228.

Taqqu, M. S., V. Teverovsky, and W. Willinger (1995), Estimators for longrange dependence: An empirical study, Fractals, 3, 785-798.

Theiler, J., S. Eubank, A. Longtin, B. Galdrikian, and D. J. Garmer (1992) Testing for nonlinearity in time-series - The method of surrogate data, Physica D, 58, 77-94.

Tziperman, E., and H. Gildor (2003), The mid-Pleistocene climate transition and the source of asymmetry between glaciation and deglaciation times, Paleoceanography, 18(1), 1001, doi:10.1029/2001PA000627.

Webster, J. M., L. Wallace, E. Silver, D. Potts, J. C. Braga, W. Renema, K. Riker-Coleman, and C. Gallup (2004), Coralgal composition of drowned carbonate platforms in the Huon Gulf, Papua New Guinea Implications for lowstand reef development and drowning, Mar. Geol., 204, 59-89.
Wunsch, C. (2003), The spectral description of climate change including the 100 ky energy, Clim. Dyn., 20, 353-363, doi:10.1007/s00382-002-0279-z.

Wunsch, C. (2004), Quantitative estimate of the Milankovitch-forced contribution to observed quaternary climate change, Quat. Sci. Rev., 23, 1001-1012, doi:10.1016/j.quascirev.2004.02.014

Yiou, P., M. Ghil, J. P. Jouzle, D. Paillard, and R. Vautard (1994), Nonlinear variability of the climatic system from singular and power spectra of late Quaternary records, Clim. Dyn., 9, 371-389.

Y. Ashkenazy, Department of Solar Energy and Environmental Physics, J. Blaustein Institute for Desert Research, Ben Gurion University, Sede Boker Campus, Midreshet Sede-Boker 84990, Israel. (ashkena@bgu.ac.il)

D. R. Baker, Earth and Planetary Sciences, McGill University, Montréal, QC, Canada H3A2A7. (donb@eps.mcgill.ca)

H. Gildor, Environmental Sciences, Weizmann Institute, Rehovot, Israel. (hezi.gildor@weizmann.ac.il) 